\title{
Special issue on automatic application tuning for HPC architectures
}

\author{
Siegfried Benkner ${ }^{\mathrm{a}, *}$, Franz Franchetti ${ }^{\mathrm{b}}$, Hans Michael Gerndt ${ }^{\mathrm{c}}$ and Jeffrey K. Hollingsworth ${ }^{\mathrm{d}}$ \\ ${ }^{a}$ University of Vienna, Vienna, Austria \\ ${ }^{\mathrm{b}}$ Carnegie Mellon University, Pittsburgh, PA, USA \\ ${ }^{\mathrm{c}}$ TU München, Munich, Germany \\ ${ }^{\mathrm{d}}$ University of Maryland, College Park, MD, USA
}

\begin{abstract}
High Performance Computing architectures have become incredibly complex and exploiting their full potential is becoming more and more challenging. As a consequence, automatic performance tuning (autotuning) of HPC applications is of growing interest and many research groups around the world are currently involved. Autotuning is still a rapidly evolving research field with many different approaches being taken. This special issue features selected papers presented at the Dagstuhl seminar on "Automatic Application Tuning for HPC Architectures" in October 2013, which brought together researchers from the areas of autotuning and performance analysis in order to exchange ideas and steer future collaborations.
\end{abstract}

High Performance Computing (HPC) systems today with a peak performance of several petaflops have hundreds of thousands of cores that have to be able to work together efficiently. Those machines have a deep hierarchy, which has to be understood by the programmer to tune his program so that it profits from higher interconnection rates. In addition, to reduce the power consumption of those systems, advanced hard- and software techniques are applied, such as dynamic voltage and frequency scaling (DVFS) or the usage of GPUs or other types of accelerators that are highly specialized for certain types of computations. This transforms a homogeneous system into a heterogeneous system, which further complicates programming and performance optimization. Due to the high amount of energy and money being lost because of low processor utilization, application developers are now investing significant time to tune their codes for current and emerging systems. Consequently, tools and techniques for facilitating an integrated performance analysis and performance tuning process on current and emerging architectures have become increasingly important.

\footnotetext{
${ }^{*}$ Corresponding author. E-mail: siegfried.benkner@univie.ac.at.
}

In the following we provide a brief overview of the five papers contained in this special issue.

"ELASTIC: A large scale dynamic tuning environment", by A. Martínez, A. Sikora, E. César and J. Sorribes presents an autotuning environment targeting large scale systems using a de-centralized approach based on a hierarchical tuning network of nodes that performs distributed analysis and performance tuning.

"Tools and methods for measuring and tuning the energy efficiency of HPC systems", by R. Schöne, J. Treibig, M.F. Dolz, C. Guillen, C. Navarrete, M. Knobloch and B. Rountree provides an overview of emerging tools and methods that deal with energy measurement and energy efficiency tuning in HPC system.

"From single- to multi-objective auto-tuning of programs: Advantages and implications", by J. Durillo and T. Fahringer reviews existing approaches to autotuning, highlighting the advantages and drawbacks of different techniques for single-objective as well as multi-objective autotuning.

"Implementation of d-Spline-based incremental performance parameter estimation method with ppOpenAT", by T. Tanaka, R. Otsuka, A. Fujii, T. Katagiri and T. Imamura proposes a method for reducing the time of automatic performance tuning by using the d-spline 
fitting function for reducing the number or necessary sampling points.

"Collective mind: Towards practical and collaborative auto-tuning”, by G. Fursin, R. Miceli, A. Lokhmotov, M. Gerndt, M. Baboulin, A.D. Malony, Z. Chamski, D. Novillo and D. Del Vento presents a col- laborative platform for tackling typical problems in autotuning, which enables the autotuning community to gather, manage and share information and knowledge with respect to all levels of autotuning. 

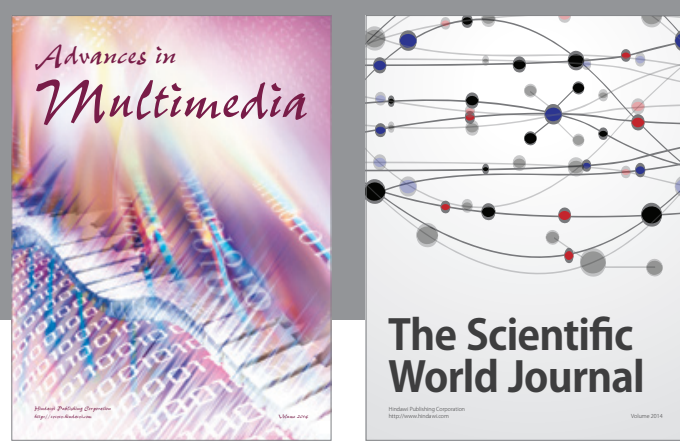

The Scientific World Journal
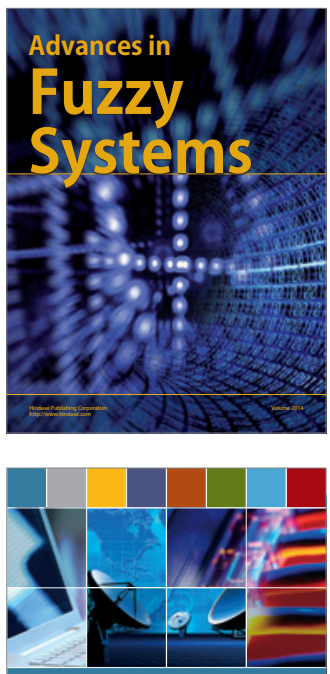

Computer Networks and Communications
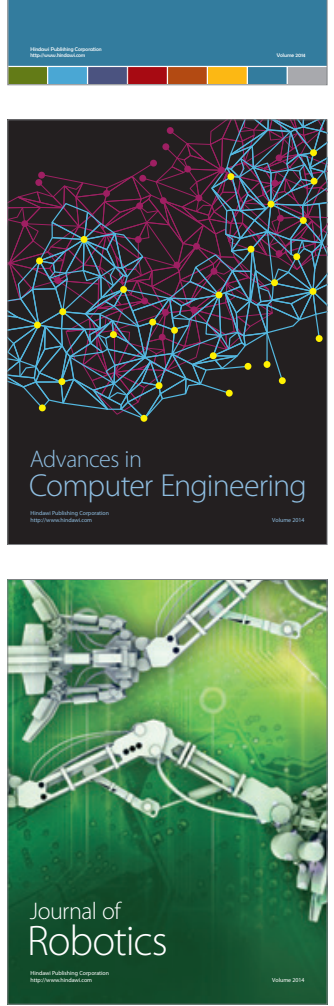
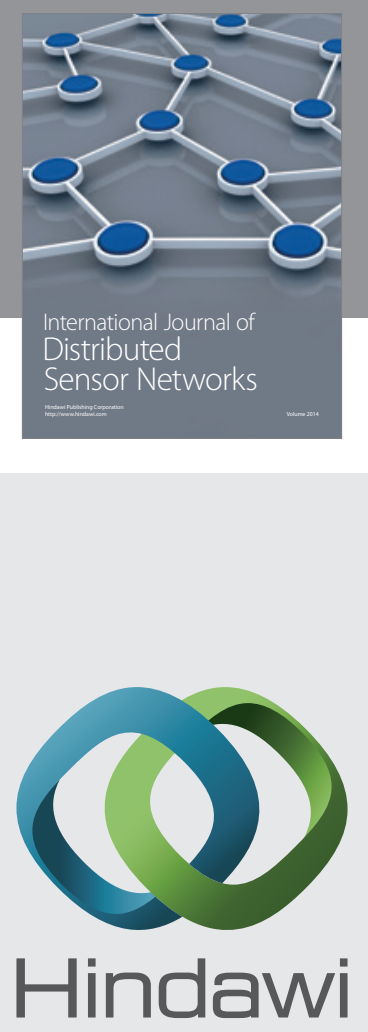

Submit your manuscripts at

http://www.hindawi.com
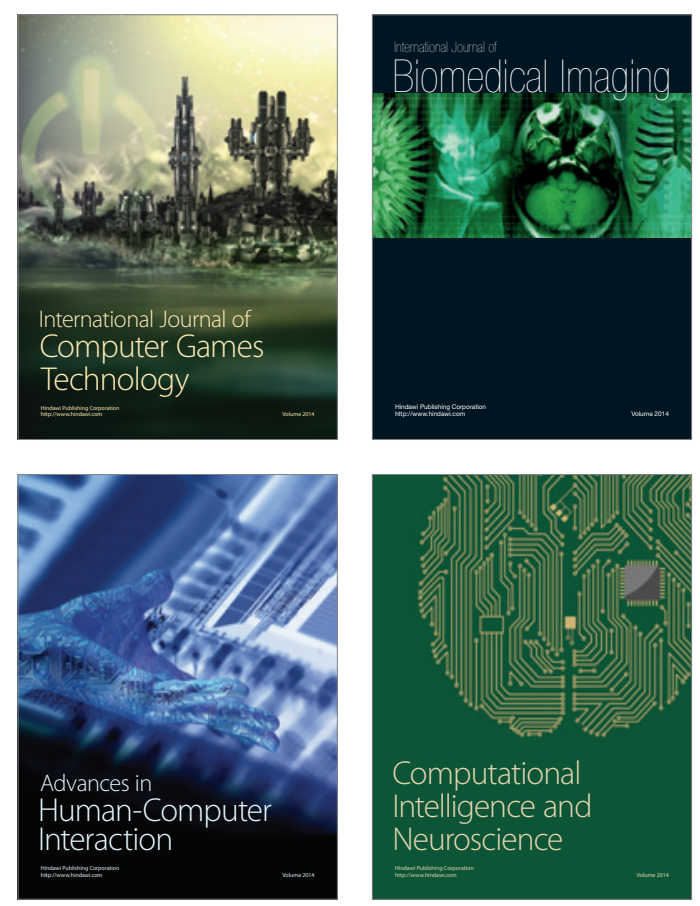
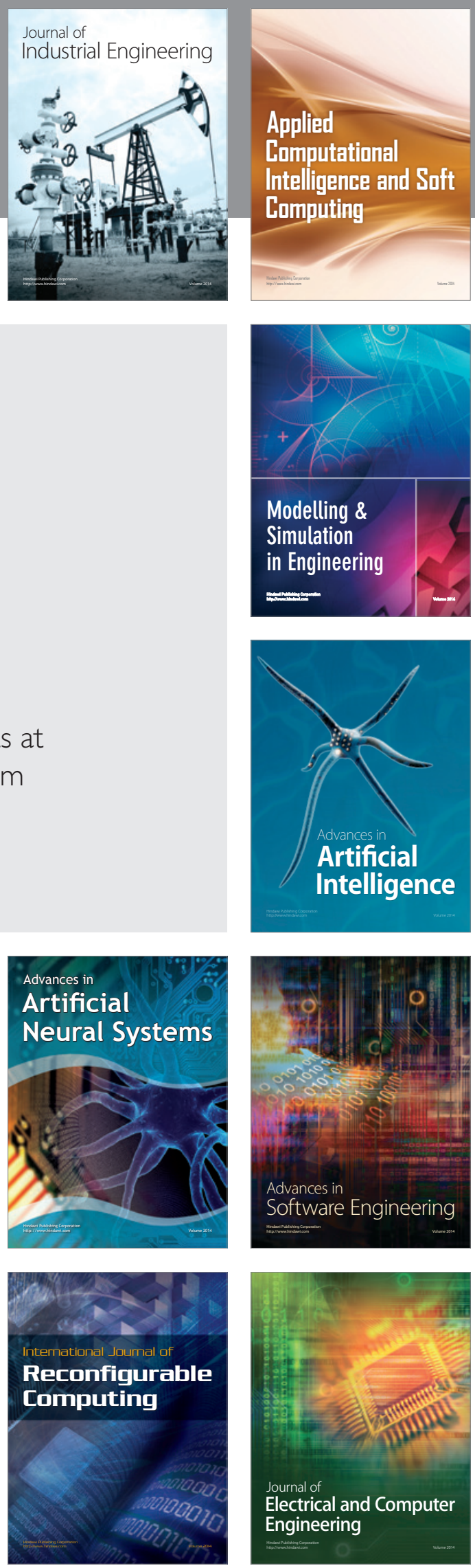